\title{
An improved simulation of the 2015 El Niño event by optimally correcting the initial conditions and model parameters in an intermediate coupled model
}

\author{
Rong-Hua Zhang ${ }^{1,2,3} \cdot$ Ling-Jiang Tao ${ }^{1,3} \cdot$ Chuan Gao $^{1,2}$
}

Received: 24 April 2017 / Accepted: 16 September 2017 / Published online: 27 September 2017

(c) The Author(s) 2017. This article is an open access publication

\begin{abstract}
Large uncertainties exist in real-time predictions of the 2015 El Niño event, which have systematic intensity biases that are strongly model-dependent. It is critically important to characterize those model biases so they can be reduced appropriately. In this study, the conditional nonlinear optimal perturbation (CNOP)-based approach was applied to an intermediate coupled model (ICM) equipped with a four-dimensional variational data assimilation technique. The CNOP-based approach was used to quantify prediction errors that can be attributed to initial conditions (ICs) and model parameters (MPs). Two key MPs were considered in the ICM: one represents the intensity of the thermocline effect, and the other represents the relative coupling intensity between the ocean and atmosphere. Two experiments were performed to illustrate the effects of error corrections, one with a standard simulation and another with an optimized simulation in which errors in the ICs and MPs derived from the CNOP-based approach were optimally corrected. The results indicate that simulations of the 2015 El Niño event can be effectively improved by using CNOP-derived error correcting. In particular, the El Niño intensity in late 2015 was adequately captured when simulations were started from early 2015. Quantitatively, the Niño3.4 SST index simulated in Dec. 2015 increased to $2.8^{\circ} \mathrm{C}$ in the optimized simulation,
\end{abstract}

Rong-Hua Zhang

rzhang@qdio.ac.cn

1 Key Laboratory of Ocean Circulation and Waves, Institute of Oceanology, Chinese Academy of Sciences, Qingdao 266071, China

2 Qingdao National Laboratory for Marine Science and Technology, Qingdao 266237, China

3 University of Chinese Academy of Sciences, Beijing 10029, China compared with only $1.5^{\circ} \mathrm{C}$ in the standard simulation. The feasibility and effectiveness of using the CNOP-based technique to improve ENSO simulations are demonstrated in the context of the 2015 El Niño event. The limitations and further applications are also discussed.

Keywords The 2015 El Niño event · ICM · The CNOPbased approach - Optimal bias corrections to ICs and MPs

\section{Introduction}

The El Niño and Southern Oscillation (ENSO) phenomenon has been recognized as the most predictable short-term climate anomaly in the climate system (Philander 1983; Wang and Picaut 2004). Predicting its onset, development and evolution accurately and effectively is of great significance to society and the public (e.g., Zebiak and Cane 1987; Chen et al. 1995, 2004; Zhang et al. 2003). Currently, various coupled models have been developed and used to make 6-month and longer real-time ENSO predictions in advance and with reasonable success. Nevertheless, coupled models with different degrees of complexity still have systematic biases with large uncertainties in the real-time prediction of ENSO (Jin et al. 2008; Luo et al. 2008; Zhang and Gao 2016b); for a summary of the model ENSO forecasts, see the International Research Institute for Climate and Society (IRI) website at http://iri.columbia.edu/climate/ENSO/currentinfo/update. html. In particular, ENSO predictions are widely spread across models, with each model having characteristic biases.

Understanding error sources for ENSO predictions is critically important for finding a way by which biases can be reduced appropriately (Blumenthal 1991; Goswami and Shukla 1991). Errors in initial conditions (ICs) and model parameters (MPs) are two main sources that limit ENSO 
prediction skills when using coupled ocean-atmosphere models (Moore and Kleeman 1996; Xue 1997). For example, based on NCEP CFSv2, Zhu et al. (2012) found that ENSO prediction skill is highly sensitive to initial states that are derived from different ocean analyses. Using an intermediate coupled model (ICM), Gao and Zhang (2017) examined the effects of the two key model parameters on depicting the intensity and phase transition for the second-year reemergence of the 2010 cold event (Zhang et al. 2013). At present, large uncertainties exist in determining ICs and MPs when using oceanic observations for ENSO predictions. Indeed, the current observations that are available in the ocean are very sparse due to their high cost and inhomogeneous distributions in space and time. Limited ocean observations therefore need to be combined with data assimilation techniques to determine ICs for model predictions. Also, observations are used to estimate model parameters and tune model performance. Nevertheless, oceanic and atmospheric models are comprehensive and very complicated, with model parameters that are strongly season and state dependent (Zhang et al. 2003, 2005). Thus, it is not easy to find suitable model parameters that allow accurate predictions. In addition, model parameters are often estimated in an empirical and subjective way; such a priori estimates of parameters may not be consistent with the model dynamics. It is desirable to estimate parameters in an optimal and objective way such that the best possible predictions can be made for a given model. Furthermore, large biases are strongly model-dependent, and the model error characteristics need to be quantified so that they can be adequately and effectively reduced.

Various approaches have been developed to find a way to characterize errors in ICs/MPs and their relationships with prediction biases. For example, Mu et al. (2003) developed a conditional nonlinear optimal perturbation (CNOP) approach to identify some types of errors in ICs that can have the largest growth. Theoretically, the CNOPs represent types of errors in ICs that can make dominant contributions to prediction biases (e.g., Mu et al. 2007; Duan et al. 2009, 2012). Practically, if the CNOP-type errors in initial states used for predictions are corrected or removed, the predictions can be improved optimally and effectively. Indeed, the CNOP approach has been extensively used to study ENSO predictability using the ENSO model developed by Zebiak and Cane (1987). For instance, Duan and Wei (2012) found that the CNOP-like errors exist in the initial fields used to make realistic ENSO predictions and thought that the ENSO forecast skill can be greatly improved if the initial analysis fields are corrected according to the CNOP-like errors. Xu (2006) and Mu et al. (2014) found the similarities between optimal precursors for ENSO and the CNOP-type errors derived in ENSO predictions. Moreover, one application of the CNOP approach is to identify sensitive areas where observations can have the strongest impacts on predictions. Duan et al. (2012) demonstrated that optimal uses of observations in the sensitive areas determined by CNOPs are not only able to reduce the effects of IC errors on predictions but also can better detect precursors of ENSO events and give relatively accurate predictions. These modeling experiments clearly demonstrate that the values of observational data for improving model predictions through ICs are dynamically different from one region to another, being geographically and seasonally dependent. Furthermore, Duan and Zhang (2010) and Mu et al. (2010) extended the CNOP approach to identify errors in MPs in an ENSO theoretical model. Subsequently, Yu et al. (2012a, b) used the Zebiak-Cane model to investigate the parametric errors that have the largest error growth in ENSO predictions. More recently, the CNOP approach has been extended to identify errors in boundary conditions that can cause the largest error growths in predictions (e.g., Wang and Mu 2015).

Previously, we developed an improved intermediate coupled model (ICM) at the Institute of Oceanology, Chinese Academy of Sciences (IOCAS), which is named IOCAS ICM (Zhang and Gao 2016a, b). The model has been routinely used to predict SST evolution in the tropical Pacific (Zhang et al. 2013). Nevertheless, pronounced biases still exist in real-time predictions of ENSO and needs to be reduced adequately. Recently, a four-dimensional variational data assimilation (4-D Var) technique has been implemented into the IOCAS ICM (Gao et al. 2016), which can provide dynamically consistent ICs for improved ENSO prediction. In addition, the adjoint component involved with the 4-D Var data assimilation technique allows the calculation of the gradient of a defined object function with respect to ICs and/ or MPs. More recently, applying the CNOP approach to the ICM, Tao et al. (2017) identified the spatial characteristics of errors in initial states that can lead to the largest growth in ENSO predictions. It was found that the CNOP-induced error evolution in the ICM exhibits a strong spring predictability barrier (SPB) phenomenon in ENSO predictions. In addition, the CNOPs derived from the IOCAS ICM provide useful information for targeted observations through ocean data assimilation. That is, when the CNOP-related errors in ICs are removed or reduced, ENSO prediction can be improved optimally and effectively. Note that these previous studies using the ICM are based on idealized experiments for ENSO predictability studies and predictions (Gao et al. 2016; Tao et al. 2017).

In this paper, we focus on a real El Niño case in 2015 to which the CNOP-based approach was applied using the IOCAS ICM. In 2015, the tropical Pacific experienced an extremely strong El Niño event, which caused significant climate anomalies and extreme weather conditions worldwide. Nevertheless, large biases existed in its real-time prediction when using various state-of-art coupled ocean-atmosphere 
models (Zhang and Gao 2016a, b). For example, a warm event was incorrectly predicted to occur in 2014 by many models, whereas the intensity of the strong El Niño event that occurred in late 2015 was significantly underestimated when predictions were made in early 2015 . Our aim here is to find a way to improve simulations of the $2015 \mathrm{El}$ Niño event by adopting the CNOP approach-based technique. Theoretically, this approach is able to characterize dominant errors in ENSO predictions that can be attributed to those in ICs and MPs, which can then be corrected for an improved simulation of the 2015 El Niño event. To this end, several steps are taken. First, an object function is defined as the differences between observed and predicted SST anomalies (i.e., SST errors in the ICM prediction). Its gradients with respect to initial fields and the MPs are calculated using the adjoint component of the 4-D Var data assimilation technique implemented in the ICM; here, two initial fields (SST and SL) and two MPs that are important to ENSO ( $\alpha_{\tau}$ and $\alpha_{\mathrm{Te}}$; Gao and Zhang 2017) are considered. The corresponding error corrections to SST, SL, and the two MPs $\left(\alpha_{\tau}\right.$ and $\left.\alpha_{\mathrm{Te}}\right)$ are then optimally quantified using the CNOP-based technique through the minimization of the object function under the constraint of model dynamics. As such, optimized error corrections to the ICs and two MPs are made such that the modeled SST evolution can be as close as possible to the corresponding observations (the object function is minimized). Finally, optimized simulations are performed with error corrections to the ICs and MPs. As will be shown below, the 2015 El Niño simulation can be effectively improved when the derived CNOP-type errors are optimally corrected in the ICM.

The paper is organized as follows. Section 2 describes the methodologies, including the ICM, 4-D Var data assimilation method, CNOP approach, and experimental designs. In Sect. 3, the extent to which the simulations of the $2015 \mathrm{El}$ Niño event can be improved by making optimal error corrections to ICs and MPs is demonstrated. Finally, a conclusion and discussion are presented in Sect. 4.

\section{Methods}

The CNOP approach is applied to an intermediate coupled model (ICM) developed by Zhang et al. (2003). The ICM has already been successfully used for ENSO simulations and predictions (Zhang and Gao 2016a). Recently, Gao et al. (2016) implemented a four-dimensional variational (4-D Var) data assimilation technique into the ICM, including the development of its adjoint component, which provides a way to calculate the gradient of a defined object function with respect to state variables and model parameters. Furthermore, the CNOP-based technique is adopted to quantify errors in ICs and MPs that can make dominant contributions to prediction biases. In an idealized model setting, Tao et al. (2017) has applied the CNOP approach to characterizing errors in ICs that can have the largest error growth in ENSO prediction (Mu et al. 2003). On the basis of those developments, the CNOP-based technique was further applied to a realistic case for the 2015 El Niño event using the ICM. Optimized corrections to errors in ICs and MPs were performed to demonstrate an improved simulation of the 2015 El Niño event.

\subsection{The ICM and its 4-D Var data assimilation technique}

The ICM used in this work is an anomaly model consisting of an intermediate ocean model (IOM) and an empirical wind stress model (Zhang and Gao 2016a). One important process for ENSO is related to the anomalous temperature of subsurface water entrained into the mixed layer $\left(\mathrm{T}_{\mathrm{e}}\right)$, which is explicitly and empirically related to sea level (SL) variation and is written as $\mathrm{T}_{\mathrm{e}}=\alpha_{\mathrm{Te}} \cdot \mathrm{F}_{1}(\mathrm{SL})$, in which $\mathrm{F}_{1}$ is a relationship between interannual variations in $T_{e}$ and SL that is determined using a singular value decomposition (SVD) analysis from historical data, and $\alpha_{\mathrm{Te}}$ is a parameter introduced to represent the strength of thermocline effect on SST (referred as the thermocline effect). A statistical atmospheric model is also constructed from an SVD analysis, in which interannual anomalies of wind stress $(\tau)$ and sea surface temperature (SST) fields are related as $\tau=\alpha_{\tau}$ $\cdot \mathrm{F}_{2}(\mathrm{SST})$, where $\alpha_{\tau}$ is an introduced parameter that represents the relative coupling intensity between the ocean and atmosphere. When taking $\alpha_{\tau}=0.87$ and $\alpha_{\mathrm{Te}}=1.0$ as a standard model setting, the ICM can well depict ENSO evolution (Zhang et al. 2003). Furthermore, a four-dimensional variation (4-D Var) data assimilation technique with the development of a adjoint component have recently been implemented in the ICM for ENSO modeling studies (Gao et al. 2016). It is shown herein that the 4-D Var method can improve ENSO simulation and prediction skill through optimal initializations of ocean initial conditions. In addition, the adjoint component involved in the 4-D Var data assimilation technique offers a way to calculate the gradient of a defined object function with respect to ICs and MPs. To improve theoretical understanding of ENSO predictability in the ICM, it is desirable to combine these ICM-based modeling studies with the CNOP approach for ENSO predictability analyses.

\subsection{The CNOP approach}

$\mathrm{Mu}$ et al. (2003) formulated the conditional nonlinear optimal perturbation (CNOP) approach, which is used to characterize errors in ICs and MPs that can make the largest contributions to prediction biases (e.g., major error source 
regions and error evolution features). Based on the ENSO model developed by Zebiak and Cane (1987), for example, this approach has been widely used to study ENSO predictability and targeted observing system designs (Xu 2006; Mu et al. 2007, 2014; Duan et al. 2009, 2012). In an idealized model setting for the IOCAS ICM, Tao et al. (2017) more recently adopted the CNOP approach (Mu et al. 2003) and theoretically demonstrated that the characterized errors in ICs can have largest error growth in ENSO predictions. A brief description of the CNOP approach is presented below.

Given prediction model, the governing equations defining an initial-value problem can be symbolically written as

$\left\{\begin{array}{l}\frac{\partial \boldsymbol{X}}{\partial t}+\boldsymbol{F}(\boldsymbol{X}, \boldsymbol{p})=0 \\ \left.\boldsymbol{X}\right|_{t=t_{0}}=\boldsymbol{X}_{0}\end{array}\right.$,

where $t$ is time, $t_{0}$ is the initial time, $\boldsymbol{X}$ is a state variable (e.g., SST and ocean currents), $\boldsymbol{p}$ is a model parameter, $\boldsymbol{X}_{\mathbf{0}}$ is an initial state of $\boldsymbol{X}$, and $\boldsymbol{F}$ is a nonlinear evolution operator. For a given initial condition $\left(\boldsymbol{X}_{\mathbf{0}}\right)$ and model parameter $(\boldsymbol{p})$, the predicted variable at time $T\left(\boldsymbol{X}^{\boldsymbol{m}}\right)$ is obtained through the time integration of the model which can be written as $\boldsymbol{X}^{\boldsymbol{m}}(T)=\boldsymbol{M}(\boldsymbol{p})\left(\boldsymbol{X}_{0}\right)(T)$, in which $\boldsymbol{M}$ is a propagator function that represents the temporal evolution from the initial state to a future state at time $T$, which is constrained by the governing equation (i.e., the model dynamics). The state variable simulated by the model at time $T$ is therefore a function of $\boldsymbol{X}_{\mathbf{0}}, \boldsymbol{p}$, and $T$. To measure the departure of the predicted evolution relative to observed state variable, an object function can be defined as

$J\left(\boldsymbol{X}_{0}, \boldsymbol{p}\right)=\sum_{t=t_{1}}^{t_{n}}\left\|\boldsymbol{X}^{\boldsymbol{m}}(t)-\boldsymbol{X}^{\boldsymbol{o}}(t)\right\|^{2}=\sum_{t=t_{1}}^{t_{n}}\left\|\boldsymbol{M}(\boldsymbol{p})\left(\boldsymbol{X}_{0}\right)(t)-\boldsymbol{X}^{\boldsymbol{o}}(t)\right\|^{2}$,

in which $t_{1}$ and $t_{n}$ are the starting and ending months for the analyses, and $\boldsymbol{X}^{\boldsymbol{o}}(\mathrm{t})$ is the observed SST anomalies. Originally, the CONP approach was used to determine initial state errors that can produce the largest growths in future evolutions $\boldsymbol{X}^{\prime}$ or predictions (Mu et al. 2003). Alternatively, this approach can be extended to quantify errors in ICs and MPs that can be responsible for the largest prediction errors. For example, due to the existence of errors in $\boldsymbol{X}_{\mathbf{0}}$ and $\boldsymbol{p}$, the evolution predicted by the model can depart from the observed evolution, leading to biases at the prediction end time and correspondingly causing increases in the object function $(J)$. To reduce errors in the predictions (and reduce $J$ ), corresponding corrections to $\boldsymbol{X}_{\mathbf{0}}$ and $\boldsymbol{p}$ can be introduced as $\boldsymbol{X}^{\prime}$ and $\boldsymbol{p}^{\prime}$, respectively. The object function is then

$J\left(\boldsymbol{X}_{0}+\boldsymbol{X}^{\prime}, \boldsymbol{p}+\boldsymbol{p}^{\prime}\right)=\sum_{t=t_{1}}^{t_{n}}\left\|\boldsymbol{M}\left(\boldsymbol{p}+\boldsymbol{p}^{\prime}\right)\left(\boldsymbol{X}_{0}+\boldsymbol{X}^{\prime}\right)(t)-\boldsymbol{X}^{\boldsymbol{o}}(t)\right\|^{2}$.
As such, the basic CNOP approach can be equivalently used to determine error corrections to ICs and MPs, by which least prediction biases can be produced when errors in the derived ICs and MPs are corrected when making predictions. In other words, the smallest departure can be achieved between optimally corrected simulations and observations. Therefore, the original CNOP-related maximization problem can be converted into a minimization problem: $\boldsymbol{X}^{\prime}$ and $\boldsymbol{p}^{\prime}$ are estimated in an optimal way such that the object function $(J)$ is minimized under the constraint of model dynamics, and the analyzed state can be as close as possible to observations.

In practice, the $\boldsymbol{X}^{\prime}$ and $\boldsymbol{p}^{\prime}$ are estimated using the CNOPbased technique, which requires the adjoint component of the original physical model (Gao et al. 2016). Because the corrected error terms $\left(\boldsymbol{X}^{\prime}\right.$ and $\left.\boldsymbol{p}^{\prime}\right)$ cannot be too large, they should be constrained to satisfy prescribed conditions, which are symbolically expressed as $\left(\boldsymbol{X}^{\prime}, \boldsymbol{p}^{\prime}\right) \in C_{\delta_{C}}$, in which $\mathrm{C}_{\delta \mathrm{c}}$ is a radius. Finally, the problem is to solve the following constrained minimization:

$J\left(\boldsymbol{X}_{0}+\boldsymbol{X}^{*}, \boldsymbol{p}+\boldsymbol{p}^{*}\right)=\min _{\left(X^{\prime}, \boldsymbol{p}^{\prime}\right) \in C_{\delta_{C}}} J\left(\boldsymbol{X}_{0}+\boldsymbol{X}^{\prime}, \boldsymbol{p}+\boldsymbol{p}^{\prime}\right) \rightarrow 0$,

in which $\boldsymbol{X}^{*}$ and $\boldsymbol{p}^{*}$ are the error correction terms to $\boldsymbol{X}_{\mathbf{0}}$ and $\boldsymbol{p}$ and are determined optimally under a given constraint condition. To minimize the object function, the adjoint component of the 4-D Var assimilation technique equipped in the ICM is used to calculate its gradients with respect to $\boldsymbol{X}^{\prime}$ and $\boldsymbol{p}^{\prime}$. To obtain $\boldsymbol{X}^{*}$ and $\boldsymbol{p}^{*}$, a nonmonotonic spectral projected gradient method (SPG2) is adopted to search for the optimal solution in the constrained problem, which, as input, needs the values of the object function and its gradient with respect to $\boldsymbol{X}^{\prime}$ and $\boldsymbol{p}^{\prime}$ and the specified constraint condition. Details on the SPG2 are given in Birgin et al. (2000). Practically, when optimally estimating $\boldsymbol{X}^{*}$ and $\boldsymbol{p}^{*}$ by minimizing the $J$, it is not necessary to modify the adjoint component in the CNOPbased analysis procedure; instead, only a change to the form of the object function is needed. Taking $\boldsymbol{X}_{\boldsymbol{0}}+\boldsymbol{X}^{*}$ and $\boldsymbol{p}+\boldsymbol{p}^{*}$ as a new corrected initial state and model parameter, the ICM is then used to perform simulations that are expected to produce the state evolutions that can be as close as possible to observations.

\subsection{Simulation experiments and the optimal correction procedure}

Figure 1 displays a schematic diagram using the ICM to show the CNOP-based analysis procedure and to demonstrate an improved simulation of the 2015 El Niño event. Detailed implementations of the CNOP approach into the ICM are presented in Tao et al. (2017). Two types of simulation experiments were performed. A standard hindcast 


\section{The CNOP-based optimized hindcast procedure}

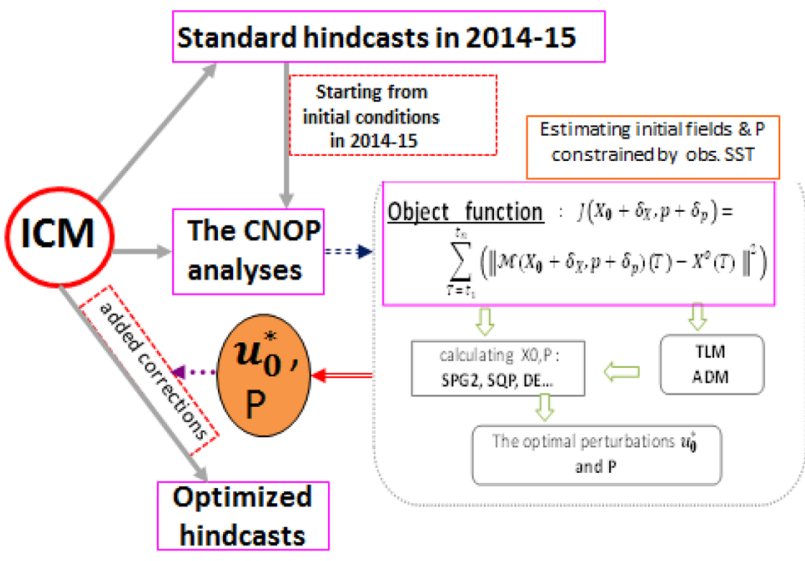

Fig. 1 A schematic diagram showing the CNOP-based analysis procedure to demonstrate an improved simulation of the 2015 El Niño event using the ICM. Three steps were taken for the demonstration. First, a standard simulation experiment was performed in which a simple initialization procedure for ICs and standard values of the two model parameters $\left(\alpha_{\mathrm{Te}}\right.$ and $\left.\alpha_{\tau}\right)$ were adopted when making predictions using the ICM. Next, the CNOP-based approach was applied to the ICM to quantify errors in predictions attributed to those in the ICs and MPs. More specifically, an object function was defined as the differences between the observed and predicted SST anomalies (SST errors in the ICM prediction); its gradients with respect to two initial fields (SST and SL) and the two MPs $\left(\alpha_{\tau}\right.$ and $\left.\alpha_{\mathrm{Te}}\right)$ were calculated using the adjoint component of the original model. Using the CNOP approach-based optimization procedure, the corresponding correction terms to the errors in SST, SL, $\alpha_{\tau}$ and $\alpha_{\mathrm{Te}}$ were optimally derived through the minimization of the object function. Finally, an optimized simulation was conducted by collectively applying the error corrections to the two initial fields (SST and SL) and the two MPs $\left(\alpha_{\mathrm{Te}}\right.$ and $\left.\alpha_{\tau}\right)$

experiment was conducted using a simple initialization procedure in which only the observed interannual SST anomaly data were used for the ENSO prediction (Zhang and Gao 2016b). Taking a prediction for 1 January 2015 as an example, a temporal succession of daily SST fields was produced using a linear interpolation method from observed monthly SST data from 1980 through December 2014 and the weekly SST data in the first week of January 2015; the observed SST data (Reynolds et al. 2002) are available online from the IRI data library. The interannual anomalies of wind stress $\left(\tau_{\text {inter }}\right)$ were derived using an empirical model of the observed SST anomalies. The derived $\tau_{\text {inter }}$ fields up to early January 2015 were used to force the ocean model to produce an initial ocean state for the first day of each month (e.g., 1 January 2015), from which predictions were made with lead times of 12 months ahead. In addition, as part of the initialization procedure, the observed SST anomalies were directly inserted into the ICM when making real-time predictions. Note that the ICM is an anomaly model in which interannual SST anomalies are directly produced. The model can well depict SST evolution. However, as shown below, the intensity of the 2015 El Niño event predicted for late 2015 was significantly underestimated when predictions were started from the initial state in late 2014 and early 2015.

The errors in the predictions can be attributed to the errors in the ICs and MPs. For example, previous studies indicate that two parameters $\left(\alpha_{\mathrm{Te}}\right.$ and $\left.\alpha_{\tau}\right)$ are important for adequately capturing the intensities of ENSO events (Gao and Zhang 2017). As detailed in the last section, the CNOPbased approach is used to determine errors in ICs and the two MPs in the ICM, which are then corrected optimally in hindcasts. Specifically, the differences between the observed and simulated SSTAs from the ICM are defined as an object function, which is written as

$J=\sum_{k=1}^{12} \sum_{i, j} \omega_{i, j}\left(\operatorname{SSTA}^{m}\left(t_{k}, i, j\right)-\operatorname{SSTA}^{o}\left(t_{k}, i, j\right)\right)^{2}$,

in which $\operatorname{SSTA}^{o}\left(t_{k}, i, j\right)$ is the monthly SSTAs observed in month $t_{k}$ at grid point $(\mathrm{i}, \mathrm{j}), \operatorname{SSTA}^{m}\left(t_{k}, i, j\right)$ is the corresponding SSTAs simulated using the ICM, and $\omega_{i, j}$ is an area-weighted function that accounts for the non-uniform horizontal grids. Because the ICM can have high skill in the central equatorial Pacific, the SST fields at grid points around the Niño3.4 region are considered in the calculation of the object function.

The constraint conditions for the error correction terms are defined as

$$
\begin{gathered}
\delta_{\mathrm{SST}}=\sqrt{\frac{\sum_{i, j} \omega_{i, j}\left[E_{\mathrm{SST}, 0}(i, j)\right]^{2}}{\sum_{i, j} \omega_{i, j}}}, \delta_{\mathrm{SL}}=\sqrt{\frac{\sum_{i, j} \omega_{i, j}\left[E_{\mathrm{SL}, 0}(i, j)\right]^{2}}{\sum_{i, j} \omega_{i, j}}} \\
\delta_{\tau}=\sqrt{\frac{1}{N} \sum_{i, j}\left[p^{\prime}{ }_{\tau}(i, j)\right]^{2}}, \text { and } \delta_{T_{e}}=\sqrt{\frac{1}{N} \sum_{i, j}\left[p^{\prime} T_{e}(i, j)\right]^{2}},
\end{gathered}
$$

in which $\mathrm{N}$ is the total grid point number of the ICM in the tropical Pacific. In practice, error correction terms are estimated in minimizing the object function with the following prescribed constraints:

$\delta_{\mathrm{SST}} \leqslant 0.1{ }^{\circ} \mathrm{C}, \delta_{\mathrm{SL}} \leqslant 0.3 \mathrm{~cm}, \delta_{\tau} \leqslant 10 \% \times \alpha_{\tau}, \delta_{\mathrm{Te}} \leqslant 10 \% \times \alpha_{T e}$.

Using the CNOP-based technique, the optimal error correction terms $\left(E_{S S T, 0}^{*}, E_{S L, 0}^{*}, p_{\tau}^{*}\right.$, and $\left.p_{T e}^{*}\right)$ can be obtained. Here, an adjoint component of the ICM is used to calculate the gradient of the object function with respect to SST, SL, $\alpha_{\tau}$ and $\alpha_{\mathrm{Te}}$, and the SPG2 approach is used to obtain these correction terms so that the object function is optimally minimized. Then, an optimal simulation is performed by applying the error corrections $\left(E_{S S T, 0}^{*}, E_{S L, 0}^{*}, p_{\tau}^{*}\right.$, and $\left.p_{T e}^{*}\right)$ to the SST, SL, $\alpha_{\tau}$ and $\alpha_{\mathrm{Te}}$ fields, respectively. Note that the 
errors in the ICs and MPs at each start month are computed simultaneously and corrected in the simulations.

We adopted seasonally varying corrections to $E_{S S T, 0}^{*}, E_{S L, 0}^{*}, p_{\tau}^{*}$, and $p_{T e}^{*}$ derived from the CNOP-based technique and conducted a series of 12-month simulations using the ICM during the period Jan. 2014-Dec. 2015. The simulation experiments for a 12-month lead-time were started from each month during 2014-2015, and $12 \times 24$ simulation results were obtained for 2014-202015. For example, when making the optimized simulations started in Jan. 2015, the correction terms derived using the CNOP-based technique were superimposed onto the originally produced initial state obtained from the standard initialization procedure, forming the corrected ICs $X_{S S T, 0}^{*}=X_{S S T, 0}+E_{S S T, 0}^{*}$ and $X_{S L, 0}^{*}=X_{S L, 0}+E_{S L, 0}^{*}$; similarly, the two MPs were corrected as $\alpha_{\tau}^{*}=\alpha_{\tau}+p_{\tau}^{*}$ and $\alpha_{T e}^{*}=\alpha_{T e}+p_{T e}^{*}$, in which $\alpha_{\tau}$ and $\alpha_{\mathrm{Te}}$ are their standard values $\left(\alpha_{\tau}=0.87\right.$, and $\left.\alpha_{\mathrm{Te}}=1.0\right)$. By applying those corrections to the corresponding ICs and MPs, the optimized simulations were performed.

As indicated in Eq. (5), the optimal analysis is performed by using observed SSTAs to form the object function which is to be minimized to obtain error corrections to ICs/MPs (the analysis period). Then, the corrected ICs/MPs are used to predict SST evolution (the prediction period). When the analysis period for minimizing the object function using observed SSTAs (e.g., 12 months) is taken the same as the prediction period, the answer (observed SSTAs) has already been put into the solution using the ICM (predicting SSTAs). In doing this, we can demonstrate the optimization procedure through which the simulated SSTAs can be depicted as close as possible to observations when making optimal error corrections to ICs/MPs using the ICM.

\section{Results}

In 2015, an extremely strong El Niño event occurred in the tropical Pacific. Figure 2 presents the Niño3.4 SST anomalies observed in 2014-2015 (black line). Figure 3 exhibits the zonal-time sections along the equator for the SST, zonal wind stress and SL anomalies, observed or reanalyzed from the atmospheric products, and Fig. 4 illustrates the corresponding horizontal distributions for SST and surface wind stress at different stages of the onset and development of the 2015 El Niño event.

A striking feature associated with that event was the slow evolution of a warm SST anomaly in the western tropical Pacific through 2014 and early 2015: a steady SST warming in the equatorial Pacific in 2014, a dip in February 2015 (the 3-month running mean SST anomaly value was below $0.5^{\circ} \mathrm{C}$ ), and a rapid warming in May that persisted through the summer and fall of 2015. A mature stage was reached in late 2015, with warm SST anomalies of $3{ }^{\circ} \mathrm{C}$ in the Niño3.4

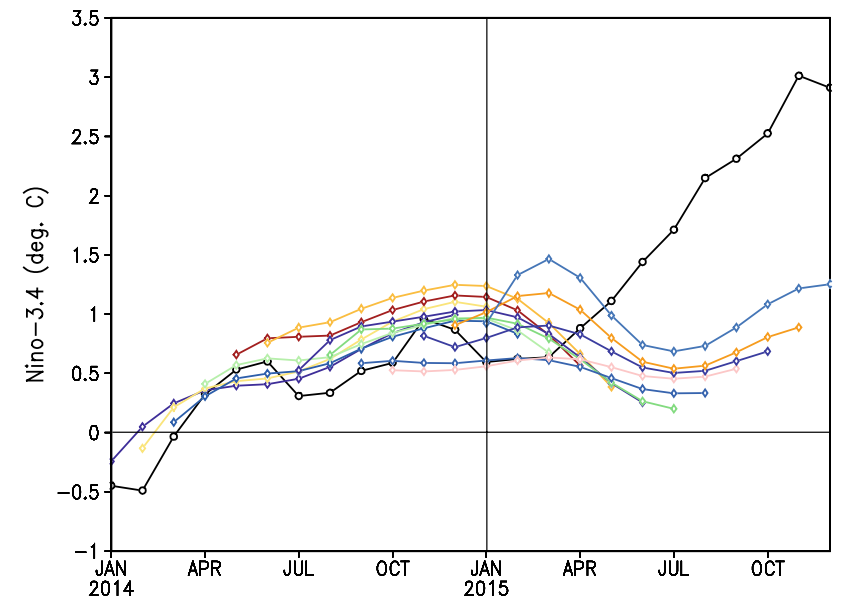

Fig. 2 The observed (black line) and predicted (colored lines) Niño3.4 SST indexes during 2014-2015 [averaged over the region $\left.\left(5^{\circ} \mathrm{S}-5^{\circ} \mathrm{N} ; 170^{\circ} \mathrm{W}-120^{\circ} \mathrm{W}\right)\right]$ from the IOCAS ICM with the original initialization scheme for the ICs and the standard values of the two MPs $\left(\alpha_{\mathrm{Te}}\right.$ and $\left.\alpha_{\tau}\right)$. Each colored line indicates a trajectory of a 12-month prediction made from different initial conditions

region in Dec. 2015. Note that the ocean and atmosphere were strongly coupled in spring 2015, upon which the warm SST anomalies amplified and developed rapidly into a warm event in the late spring of 2015. Herein, this latest $2015 \mathrm{El}$ Niño event is used as a test bed to demonstrate the feasibility and effectiveness of using the CNOP-based technique to improve its simulations by performing optimal corrections to errors in ICs and the two MPs. Two experiments were designed using the ICM, with a 12-month simulation being conducted for each month during the periods from Jan. 2014 to Dec. 2015.

\subsection{A standard simulation}

Figure 2 displays the Niño3.4 SST anomalies predicted using the ICM started from different initial times during the periods 2014-2015 (colored lines). For this standard simulation experiment, a simple initialization scheme was adopted (Zhang and Gao 2016b) in which observed interannual SST anomalies were used to derive an interannual wind stress $(\tau)$ field that was used to force the ocean model to yield initial ocean conditions, and the two MPs were taken as their standard values $\left(\alpha_{\mathrm{Te}}=1.0\right.$ and $\left.\alpha_{\tau}=0.87\right)$. Although the ICM can depict the general warming tendency in 2014-2015, obvious discrepancies existed between the observed and predicted SST anomalies. In particular, the predicted intensity of the 2015 El Niño event was significantly underestimated when predictions were started from early 2015. As with the socalled spring predictability barrier phenomenon, the model predictions indicated poor performance when predictions were made through spring 2015. In addition, the model had 
(a) SSTA

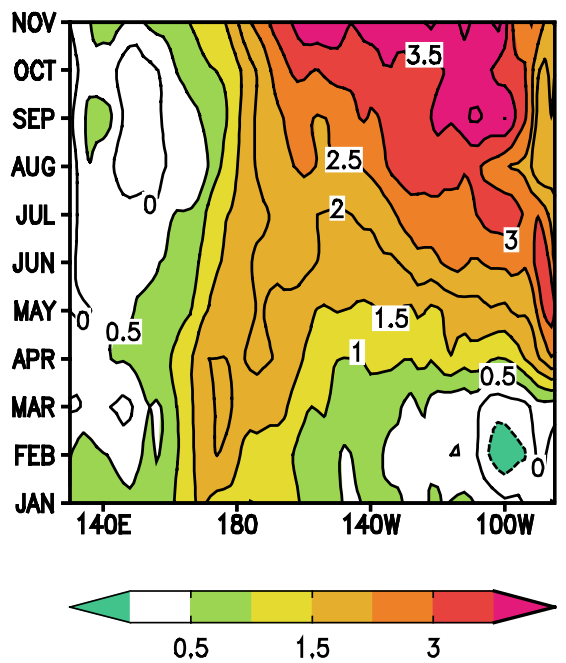

(b) Zonal wind stress

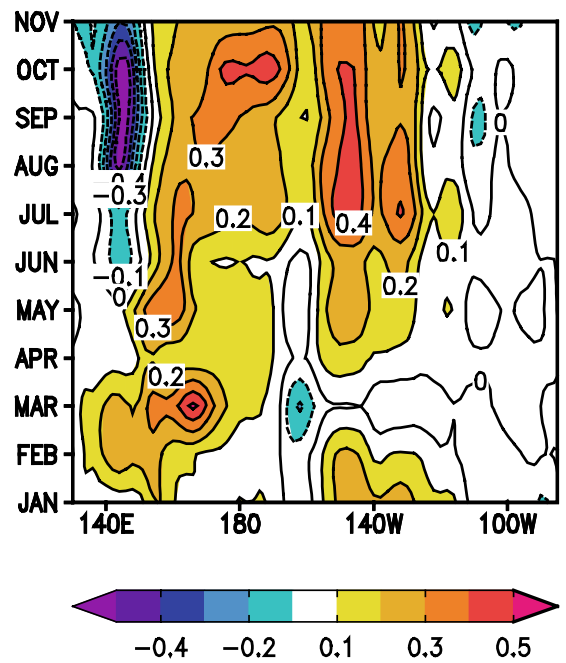

(c) SLA

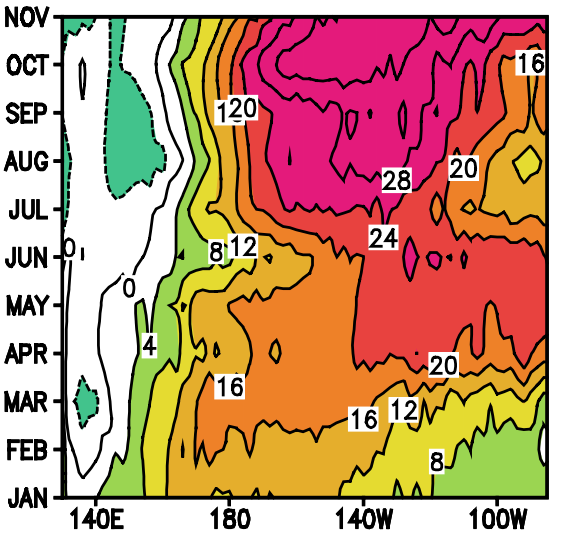

Fig. 3 Zonal-time sections along the equator for a SST, b zonal wind stress, and $\mathbf{c}$ SL anomalies. The SST fields are observed from Reynolds and Smith (1994); the wind stress fields are from the NCEP/ NCAR reanalysis (Kalnay et al. 1996); the gridded sea level anoma- lies are from the Ssalto/Duacs multimission altimeter products distributed by Aviso with support from Cnes, which are available online at http://www.aviso.oceanobs.com/duacs/. The contour intervals are $0.5^{\circ} \mathrm{C}$ for SST, $0.1 \mathrm{dyn}^{-2}$ for wind stress and $4 \mathrm{~cm}$ for SL (a) Feb

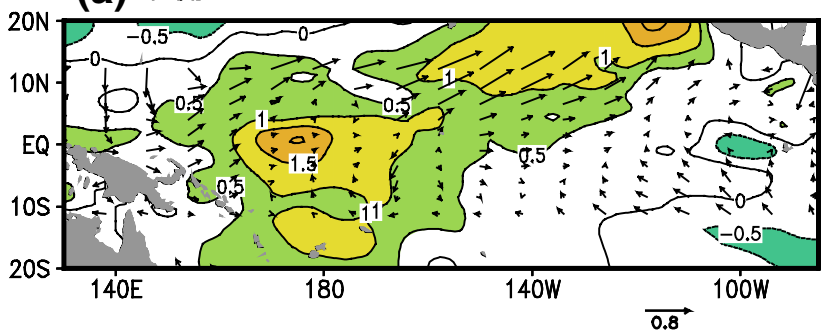

(b) Apr

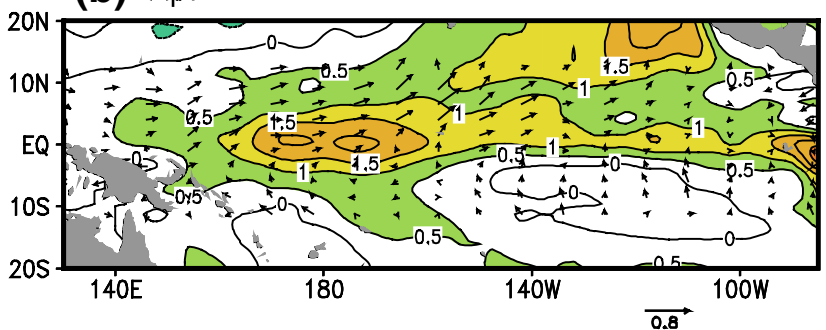

(c) Jun

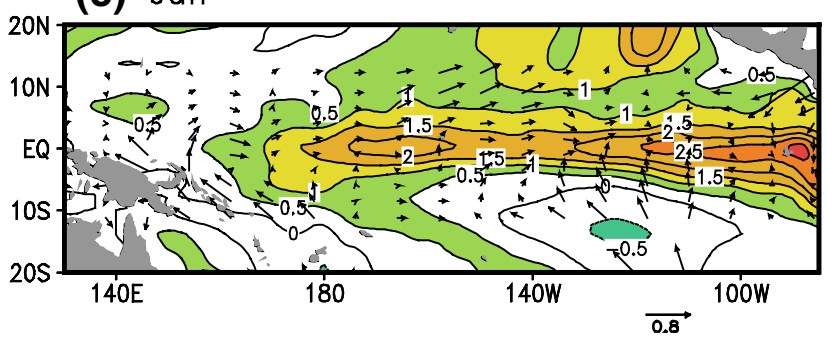

Fig. 4 Horizontal distributions of SST anomalies (contours) and wind stress anomalies (vectors): a Feb. 2015, b Apr. 2015, c Jun. 2015, d Aug. 2015, e Oct. 2015, and f Dec. 2015. The SST fields are (d) Aug

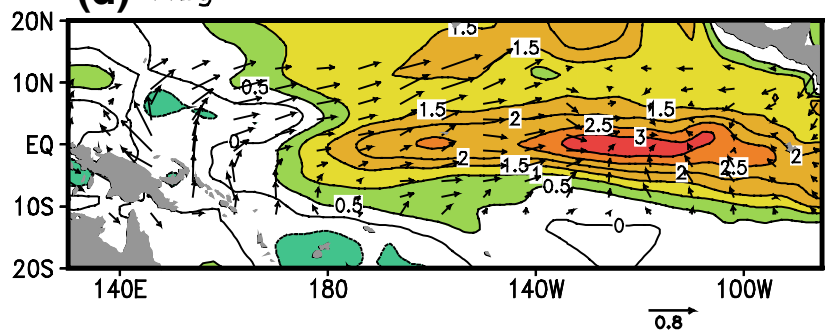

(e) Oct

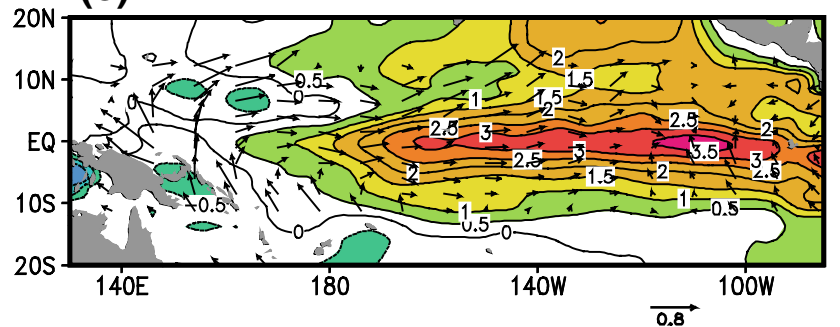

(f) $\mathrm{Dec}$

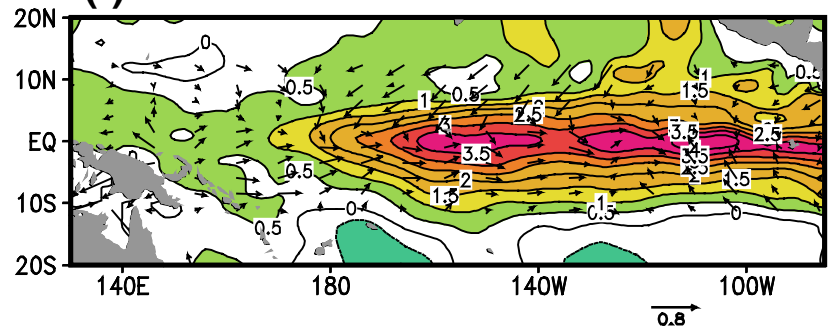

from Reynolds and Smith (1994) and the wind stress data are from the NCEP/NCAR reanalysis (Kalnay et al. 1996). The contour intervals are $0.5^{\circ} \mathrm{C}$ for SST 
difficulty predicting the rapid warming during the summer and fall of 2015 when the predictions were initialized in early 2015. As a result, the warming intensity for the 2015 El Niño event was underestimated in the summer and fall of 2015. Nevertheless, starting from the late summer of 2015, the model was able to adequately capture the warm conditions in late 2015 (Zhang and Gao 2016b).

To illustrate the space-time evolution in more detail, Fig. 5 exhibits the zonal-time sections along the equator for the SST, zonal wind stress and SL anomalies predicted from the initial conditions on 1 Jan. 2015. In addition, snapshots of the corresponding horizontal distributions for SST and surface wind stress at different stages of the onset and development are illustrated in Fig. 6; these maps start with the El Niño onset in Jan. 2015 and peak El Niño conditions in late 2015.

The model indicates obvious discrepancies in predicting the 2015 El Niño event. For example, the model had difficulty depicting the onset and development of the $2015 \mathrm{El}$ Niño event when initialized in late 2014. The model also had difficulty in predicting the rapid warming that occurred in the late spring and summer of 2015. In particular, the predicted SSTA exhibited a decreased warming tendency when the predictions were started in early 2015. As a result, the warming intensity in the summer and fall of 2015 was significantly underestimated. This indicates that the model has problems in adequately capturing the coupling intensity between the ocean and atmosphere, which can be related to the two key model parameters.

Other coupled models used to make real-time ENSO predictions have produced similar discrepancies in predictions of the 2015 El Niño event (Zhang and Gao 2016b). For example, many coupled models experienced obvious problems in adequately predicting the onset in the spring of 2015 and the rapid warming during the late spring and summer of 2015 when predictions were initialized in early 2015 (see the IRI website at http://iri.columbia.edu/climate/ENSO/ currentinfo/update.html). In addition, most of the coupled models tended to underestimate the warming intensity during the summer and fall of 2015. Additionally, the predicted intensity of the $2015 \mathrm{El}$ Niño event exhibited a wide range across various coupled models.

\subsection{An optimal simulation}

As detailed above, the CNOP-based technique was used to characterize errors in the ICs and MPs, which were then optimally corrected. Another optimal simulation experiment was therefore performed such that at the start time of the simulations, optimal corrections derived using the CNOP technique were applied to the errors in $E_{S S T, 0}^{*}, E_{S L, 0}^{*}, p_{\tau}^{*}$, and $p_{T e}^{*}$. Twelve-month simulations were then performed for each month during the periods 2014-2015. We pursued the following questions. How well can the defined object function be minimized when performing error corrections to the ICs and MPs in the ICM? To what extent can the 2015 El Niño event simulation be improved using the CNOP-based optimal corrections to the errors in the ICs and MPs?

As defined in Sect. 3, the object function represents the departures between the predicted and observed SST anomalies. The adjoint component of the 4-D Var data assimilation method implemented in the ICM was used to calculate its gradient with respect to the two initial states (SST and SL) and the two MPs $\left(\alpha_{\mathrm{Te}}\right.$ and $\left.\alpha_{\tau}\right)$ for minimizing the object (a) SSTA

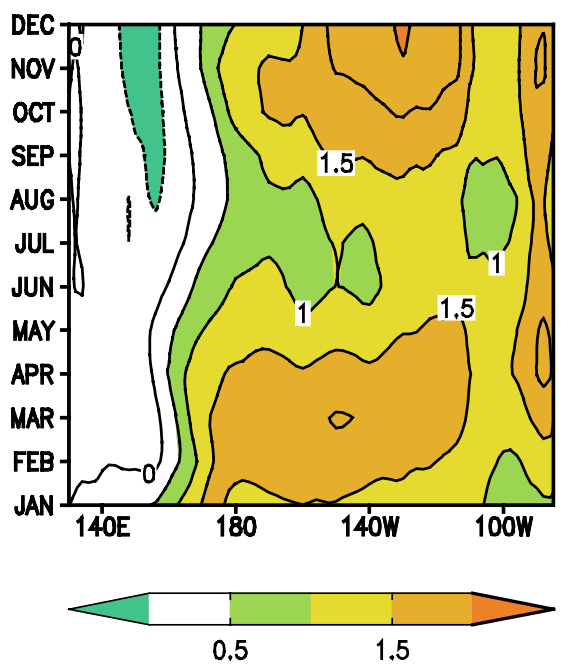

(b) Zonal wind stress

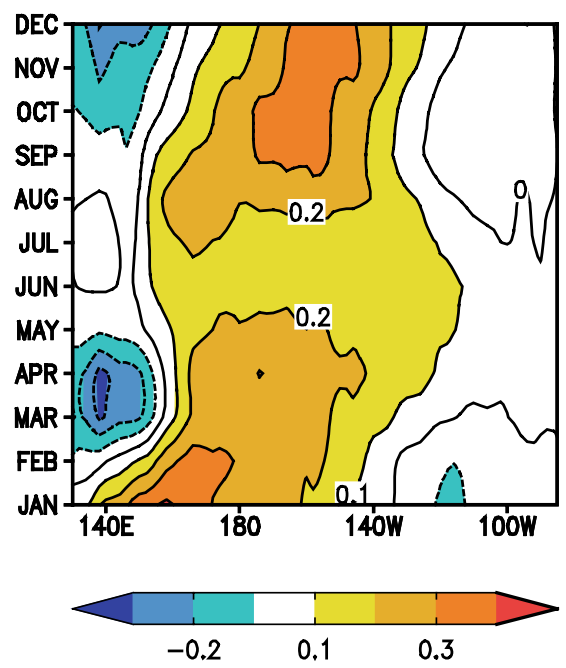

(c) SLA

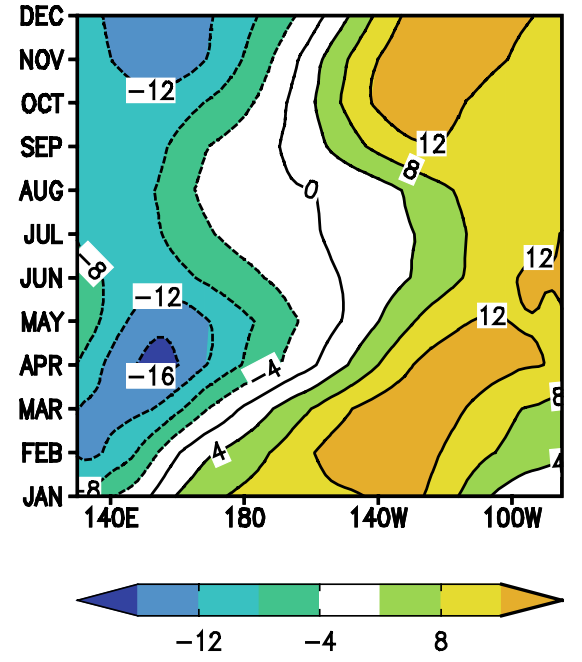

Fig. 5 Zonal-time sections along the equator for a SST, b zonal wind stress, and $\mathbf{c}$ SL anomalies, which were predicted from the initial condition on 1 Jan. 2015 for the standard hindcast. The contour intervals are $0.5^{\circ} \mathrm{C}$ for SST, 0.1 dyn $\mathrm{cm}^{-2}$ for wind stress and $4 \mathrm{~cm}$ for SL 
(a) Feb

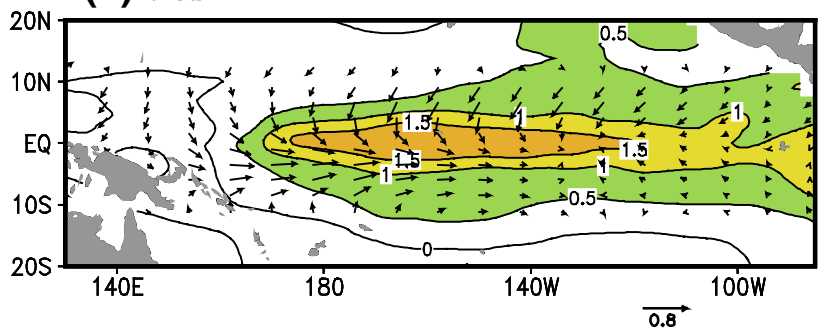

(b) Apr

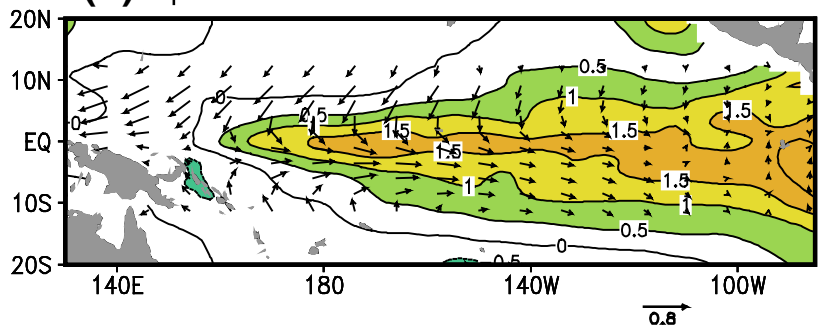

(c) Jun

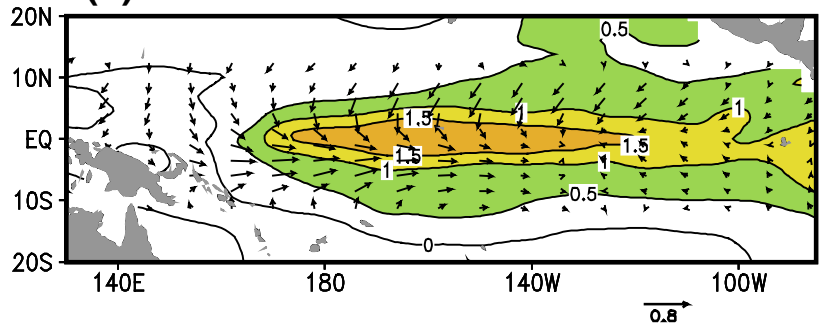

Fig. 6 Horizontal distributions of SST anomalies (contours) and wind stress anomalies (vectors) for the standard hindcast made from the initial conditions on 1 Jan. 2015: a Feb. 2015, b Apr. 2015, c Jun.

function. The errors in the ICs and MPs that could cause the largest prediction biases were quantified and then optimally corrected when performing the simulation. Figure 7 displays the spatial distributions of the correction terms to the errors in SST, SL, $\alpha_{\mathrm{Te}}$ and $\alpha_{\tau}$, which were derived for simulations started from Jan. 2015. The regions with large corrections to the errors in the ICs were consistent with the sensitive regions derived using the CNOP-based approach, where the errors in ICs can cause the largest prediction errors (Tao et al. 2017). In addition, notable corrections were seen for the two parameters, indicating that they should be taken as spatially varying. As a measure of the success in minimizing the discrepancies between the model solutions and observations, Fig. 8 further illustrates the time series of the object function estimated as a function of start time when performing the simulations. A reduction was clearly seen in the object function when performing the CNOP-based analyses, indicating that the misfit between simulated and observed SST anomalies was decreasing when the minimization was executed.

Figure 9 illustrates the Niño3.4 SST anomalies produced from the optimized simulations. When those errors in the (d) Aug

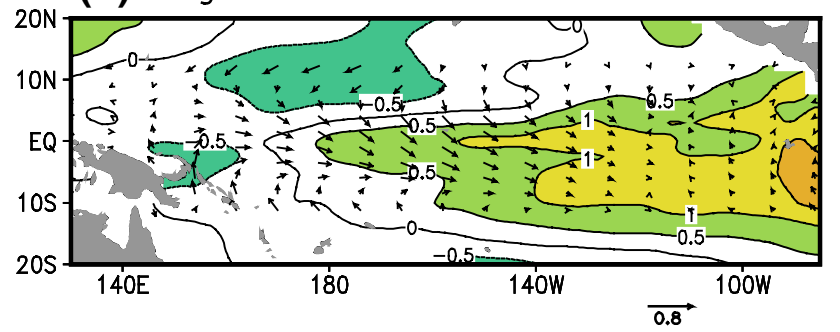

(e) Oct

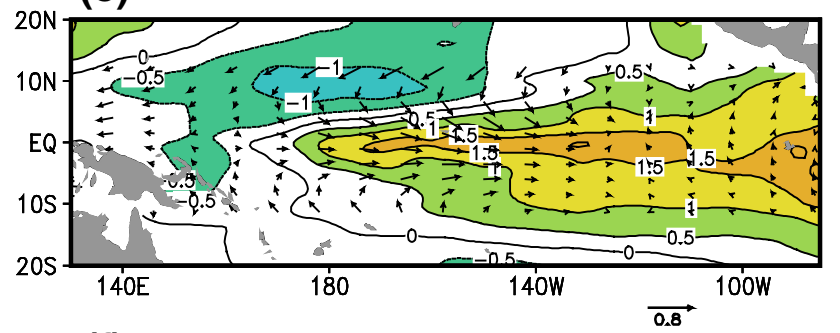

(f) $\mathrm{Dec}$

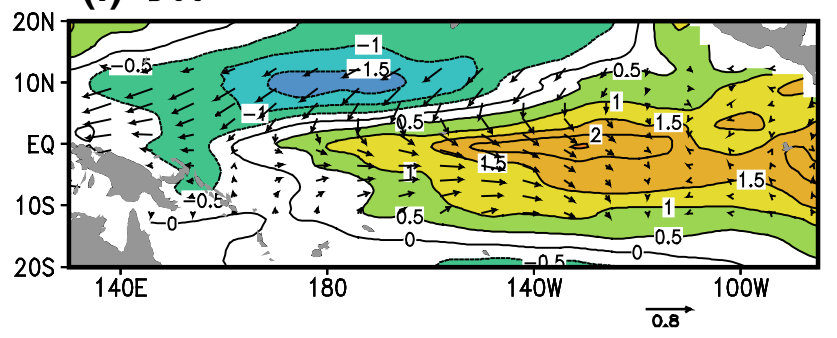

2015, d Aug. 2015, e Oct. 2015, and f Dec. 2015. The contour intervals are $0.5^{\circ} \mathrm{C}$ for SST

ICs and MPs were optimally corrected, the simulated SST anomalies closely followed those observed. Even starting in Jan. 2015, the model was able to accurately depict the sustained warming, which peaked in fall 2015 and developed into a mature phase in late 2015. Quantitatively, the produced Niño3.4 SST index was increased from $1.5^{\circ} \mathrm{C}$ in the standard simulation case to $2.8^{\circ} \mathrm{C}$ in the optimally corrected case.

Detailed comparisons can be made between the optimized simulations (Figs. 9, 10, 11) and the standard simulations (Figs. 2, 5, 6). The performances of the model simulations are shown during various stages-onset, development and mature-of the $2015 \mathrm{El}$ Niño event. When the corrected ICs and two MPs derived from the CNOP-based technique were used to perform simulations in the ICM, the model ably captured the SST evolution when the simulations were started from early 2015 (Figs. 9, 10, 11) when compared with the results of the standard simulations (Figs. 2, 5, 6). The obvious discrepancies seen in the standard simulations disappeared in the optimal simulations. For example, the warming intensity in the summer and fall of 2015 was adequately captured when the simulations were started from 
(a) $\mathrm{SSTA} ; \mathrm{CR}=0.02^{\circ} \mathrm{C}$

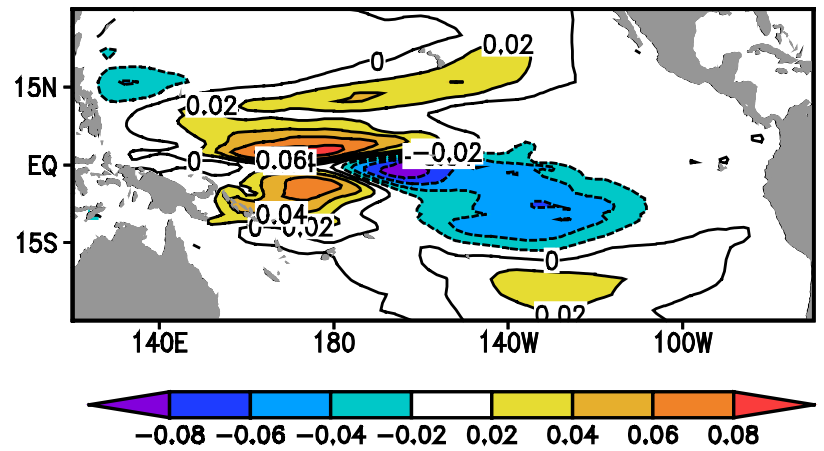

(c) $\alpha_{r} ; C R=0.087$

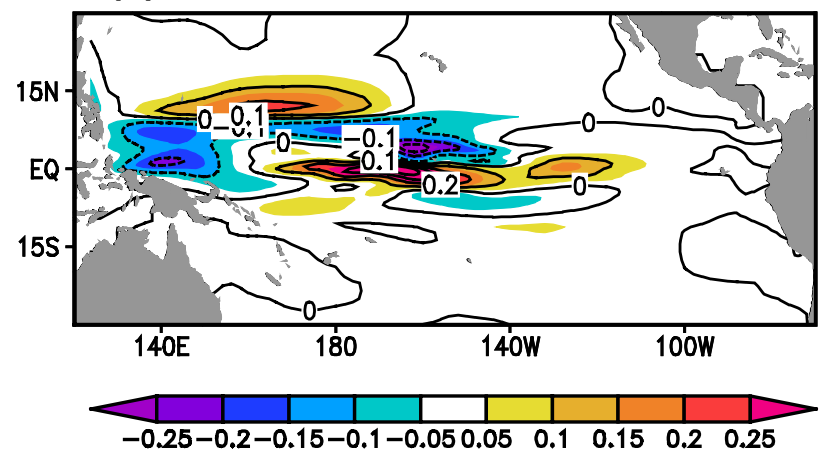

(b) $\mathrm{SLA} ; \mathrm{CR}=0.11 \mathrm{~cm}$

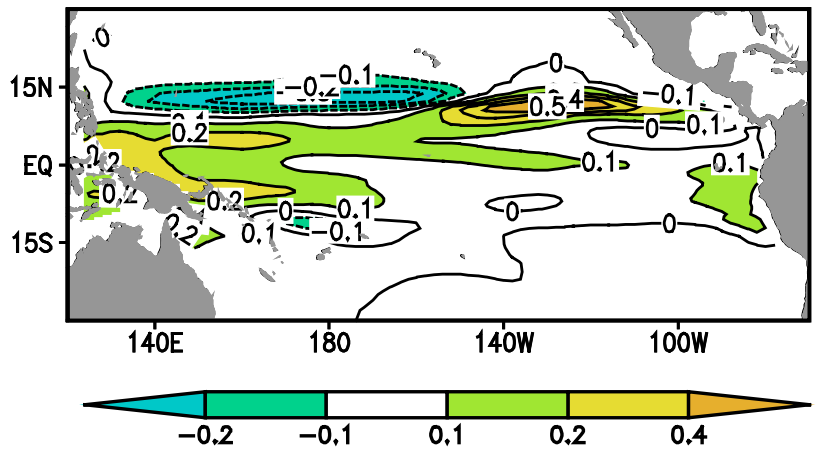

(d) $\alpha_{\mathrm{Te}} ; \mathrm{CR}=0.1$

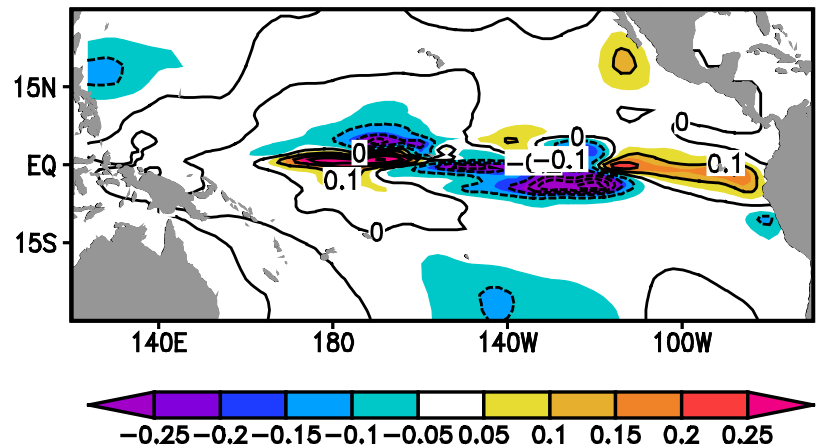

Fig. 7 Spatial distributions of the correction terms to errors in a SST, $\mathbf{b}$ SL, $\mathbf{c} \alpha_{\tau}$ and $\mathbf{d} \alpha_{\mathrm{Te}}$, which were derived using the CNOP approach-based technique for simulations started from Jan. 2015

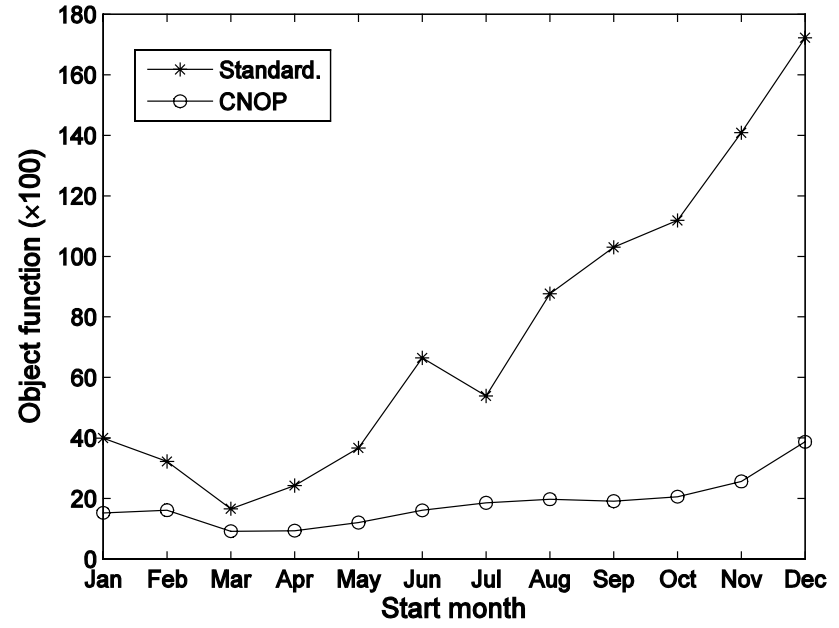

Fig. 8 Time series of the object function estimated as a function of target time when performing simulations from Jan. 2015. The adjoint component of the 4-D Var data assimilation method implemented in the ICM was used to calculate the gradient of the object function with respect to the two initial states (SST and SL) and the two MPs $\left(\alpha_{\mathrm{Te}}\right.$ and $\alpha_{\tau}$ )

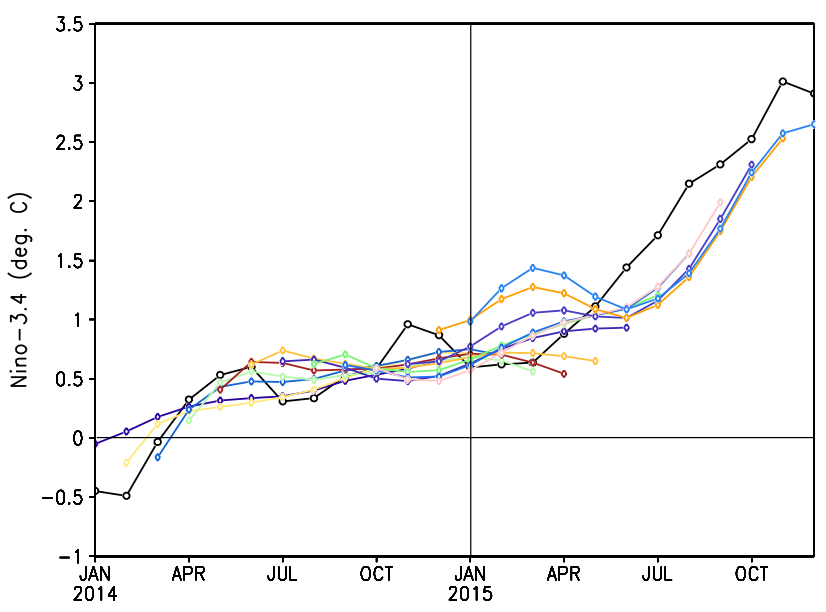

Fig. 9 The same as in Fig. 2 but for the optimized simulation (i.e., optimal corrections were performed to the errors in SST, SL, $\alpha_{\tau}$ and $\alpha_{\mathrm{Te}}$ which were quantified using the CNOP approach-based technique) 
(a) SSTA

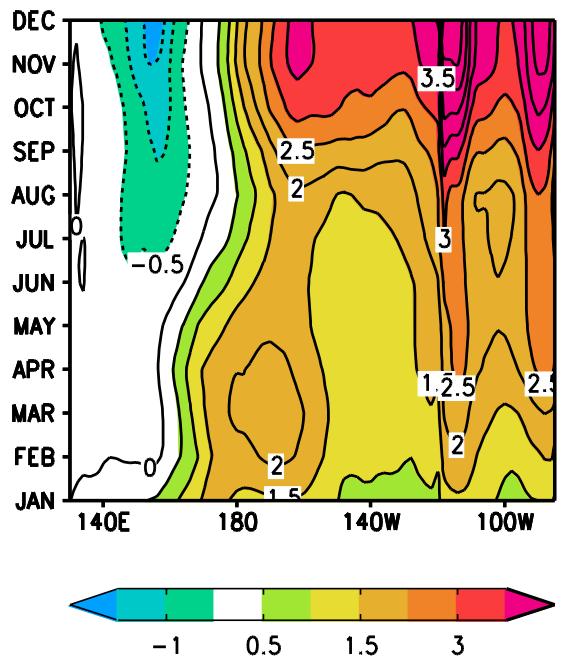

(b) Zonal wind stress

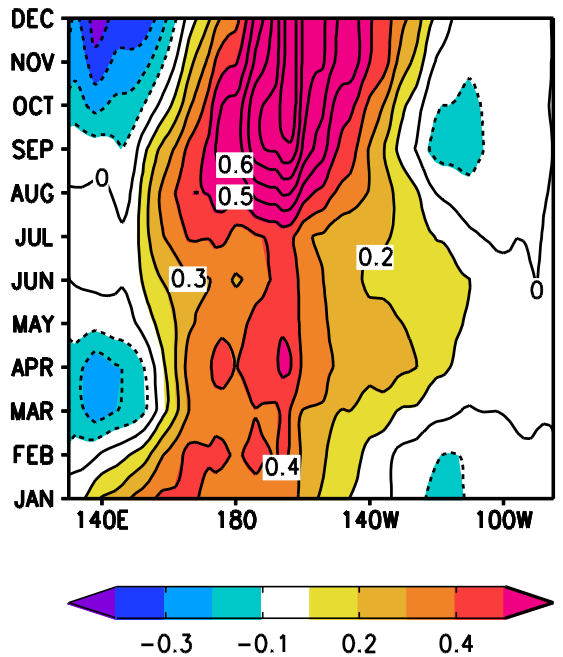

(c) SLA

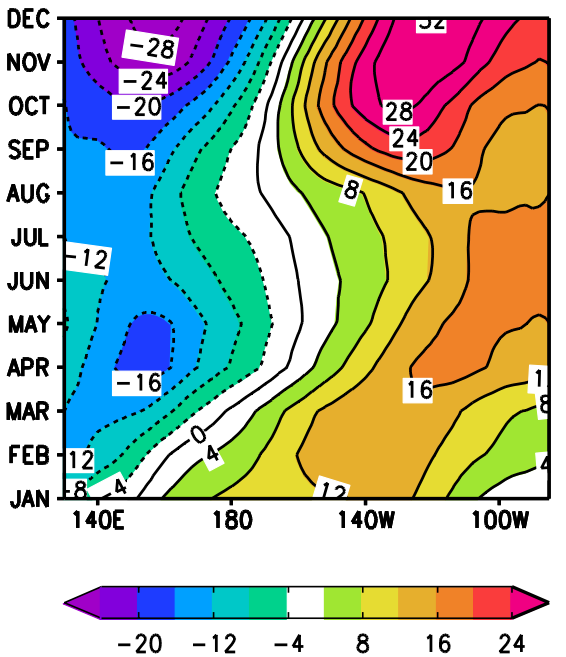

Fig. 10 The same as in Fig. 5 but for the optimized simulation

(a) Feb

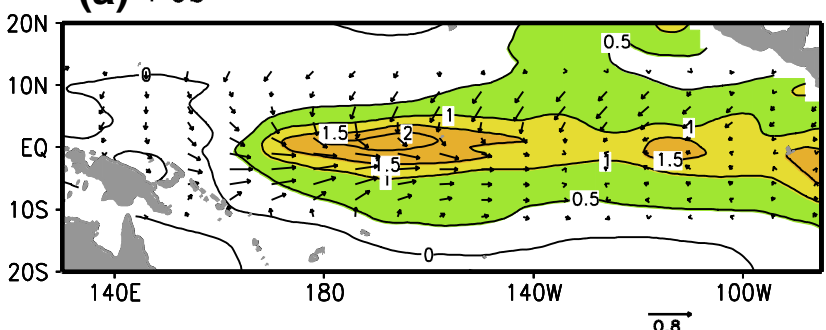

(b) Apr

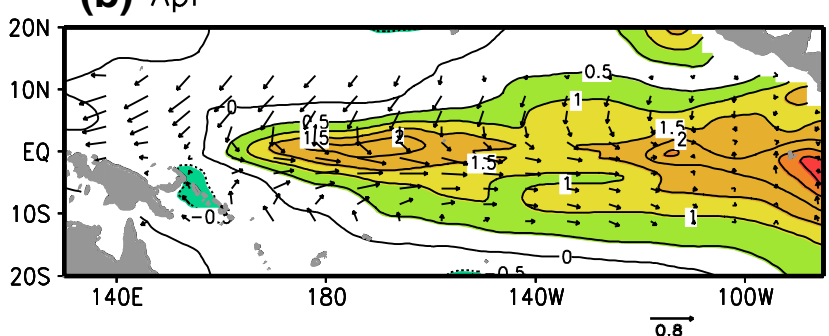

(c) Jun

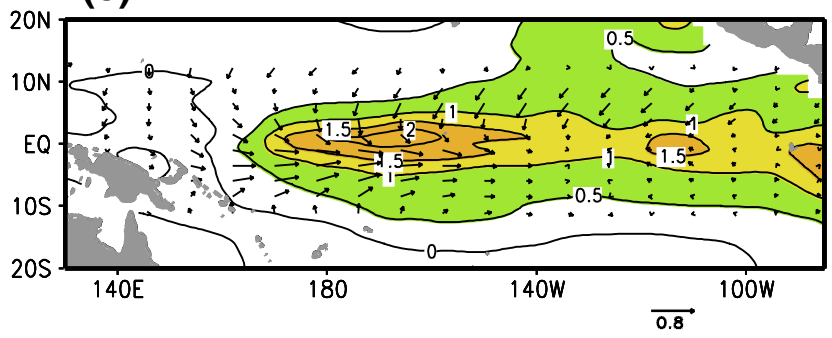

Fig. 11 The same as in Fig. 6 but for the optimized simulation

early 2015 (Fig. 9). The rapid amplification in late spring was adequately captured when the optimal corrections were made to the errors in the ICs and MPs. Subsequently, the (d) Aug

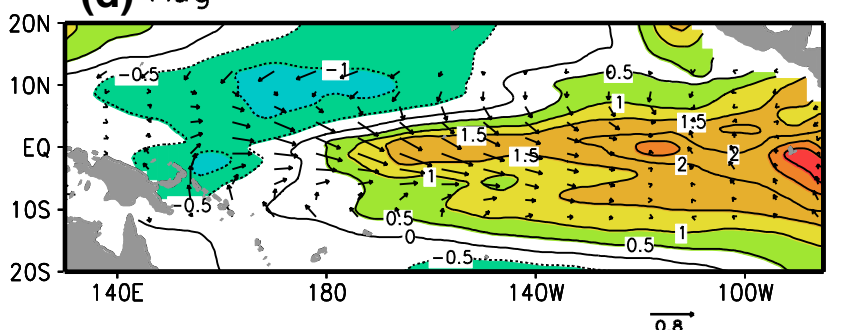

(e) Oct

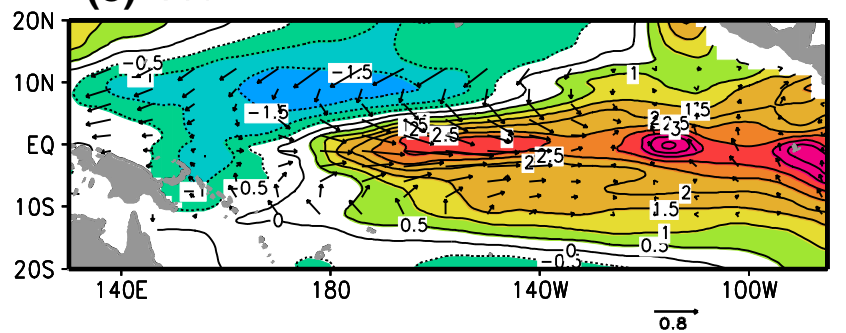

(f) $\mathrm{Dec}$

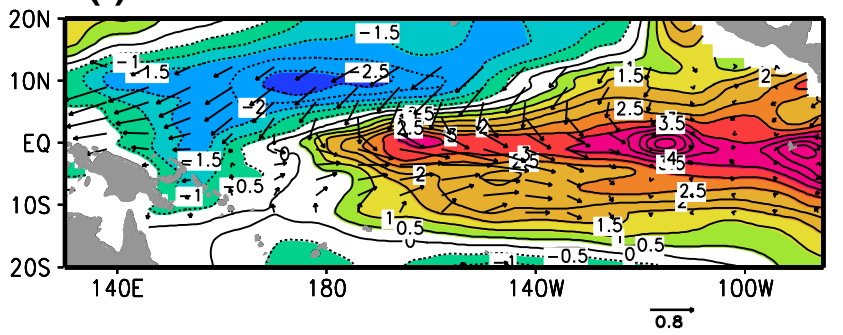

warm SST anomalies amplified and developed rapidly into a strong warm event in summer 2015.

It is straightforward to explain the improvements seen in the optimized simulations (Figs. 9, 10, 11) compared with 
the standard simulations (Figs. 2, 5, 6). Theoretically, the CNOP approach allowed the errors to be quantified in the ICs and MPs that could cause the largest error growth in the ENSO prediction. The errors in the ICs and MPs were then optimally corrected, and the related error growths were effectively inhibited. It is not surprising that simulation biases can be reduced. In addition, those two parameters represent the Bjerknes feedback (Bjerknes 1969), which involves the couplings among the surface winds, SSTs and thermocline fluctuations that are important to ENSO development. The corrections to the errors in the two MPs led to an optimal representation of the two processes involved: the thermocline effect on SST and the relative coupling between the ocean and atmosphere. For example, $\alpha_{\mathrm{Te}}$ is part of the Bjerknes feedback that is related to the thermocline's role in ENSO evolution; its value represents the intensity of the direct thermocline effect on SST. When the intensities of these processes are represented in an optimal way, it is therefore expected that simulations can be adequately improved. As numerically justified above, optimized error corrections to the ICs and two MPs led to the improved simulations of SST evolution in 2015. As such, a best possible model simulation can be obtained in the sense that error corrections to ICs and MPs derived from the CNOP approach-based technique are required for the ICM to give rise to the smallest departures between simulated and observed SSTAs.

\section{Conclusion and discussion}

The tropical Pacific experienced a sustained warming condition during 2014-2015. In particular, following the warm SSTs throughout 2014, a strong El Niño event developed in 2015. Its onset occurred in early 2015 , a rapid amplification of the warm SST anomalies occurred in late spring and summer, and a mature stage was reached in late 2015 (Zhang and Gao 2017). Various state-of-the-art coupled models have been routinely used to make ENSO predictions, but systematic biases exist in those real-time predictions which were widely spread across the various coupled models. In particular, the intensity of the 2015 El Niño event was significantly underestimated when predictions were started from early 2015. It is critically important to identify error characteristics so that predictions can be adequately improved. These prediction biases can be attributed to many factors, including errors in ICs and MPs. The previously developed CNOP approach can be used to quantify errors in ICs/MPs and their relationships with prediction biases. Naturally, if errors in ICs and MPs are derived using the CNOP approach and are then optimally corrected, it is expected that prediction biases can be reduced with the achieved improvements. These ideas have been tested in a real case for the 2015 El Niño event.
Such a reduction in model biases is demonstrated by performing two types of simulations using the IOCAS ICM. In a standard simulation, the model is initiated using a simple initialization procedure. The model can predict the $2015 \mathrm{El}$ Niño event to some extent, but the intensity of the event is significantly underestimated when predictions are started from early 2015 through spring 2015 . An optimized simulation was then conducted in which errors in the ICs and MPs derived from the CNOP-based technique were corrected when performing simulations. These results indicate that the CNOP approach can be effectively used to improve simulations due to its ability to quantify errors in ICs and MPs, which can then be optimally corrected. The simulations were significantly improved, including the intensity of the 2015 El Niño event in late 2015 when the simulations were performed in early 2015 . Herein, a modeling framework was presented to clearly demonstrate the feasibility and effectiveness of using the CNOP-based approach to optimally correct errors in ICs and MPs to improve ENSO simulations. The idea is tested as a case study and attempted to improve the 2015 El Niño simulation skill in the ICM.

This study adopted the basic idea of the CNOP approach to optimally quantify the error corrections to ICs and MPs. Theoretically, the CNOPs represent a kind of perturbations to ICs/MPs which can have the largest error growth in model prediction. Mathematically, an object function is defined as the departures between SST anomalies predicted and observed, and its minimization is solved through the CNOP approach-based optimization procedure, in which optimal error corrections to ICs and MPs are quantified using the adjoint component of the original model. Then, making error corrections to ICs/MPs can give rise to the smallest errors in prediction (i.e., simulated SSTAs can be as close as possible to observations). Note that the optimization of the ICs, as well as the MPs can also be accomplished using a standard 4-D Var data assimilation alone (Gao et al. 2016). As described above, the minimization of the object function using the CNOP approach-based technique is more or less similar to what the 4-D Var data assimilation does. Here, we adopt the CNOP-based technique to form a constrained minimization problem of an object function for quantifying optimal error corrections (Mu et al. 2003) and at the same time use the adjoint component of the original model to calculate the gradients of the object function with respect to ICs/MPs (Gao et al. 2016). Being distinguished from the standard 4-D Var data assimilation, one role played by the CNOP approach-based technique is the constraint conditions imposed on ICs/MPs when forming the minimization problem of the object function for finding an optimal solution. Then, the nonmonotonic spectral projected gradient method (SPG2) is adopted to search for the optimal solution in the constrained problem (Birgin et al. 2000). This is the 
main value which is added in this procedure when the CNOP approach-based technique is adopted here.

In this study, the analysis window with which observed SSTAs are used to define the object function in the CNOP approach-based optimization is taken as 12 months, the same period as the hindcast is made. In so doing, the answer (the observed SSTAs) has already provided to the solution (predicting SSTAs using the model). Taking the same analysis period as the prediction period means that the CNOP-based analysis only has diagnostic value, but not useful in the prediction sense. Note that if the analysis period (say taking 6 months) is chosen to be shorter than that the prediction period (say taking 12 months), predictions for some months ahead can be made beyond the analysis period so that the optimized ICs/MPs can have predictive values. Modeling experiments will be performed to demonstrate this in the future.

Also, in this paper, we only present a case study for the 2015 El Niño event. When the procedure is applied to the 2015 El Niño event, good results are obtained, but the simulation could be over-tuned for the model to make a big El Niño. The procedure can be easily applied to other events. Actually, we have also examined the effectiveness of these error corrections to other El Niño events with similar success. Additionally, it is desirable to examine the extent to which the optimized model parameters derived from 2015 are useful for the prediction of other cases (e.g., the 1997-1998 event). Further tests and applications in these lines are underway and will be presented elsewhere.

Acknowledgements We would like to thank Drs. Qiang Wang, Wansuo Duan, $\mathrm{Mu} \mathrm{Mu}$, Xinrong Wu, and Hui Xu for their comments and helps in implementing the CNOP approach into the IOCAS ICM. We also wish to thank three anonymous reviewers for their insightful comments and constructive suggestions. This research was supported by the National Natural Science Foundation of China (Grant nos. 41690122, 41690120), AoShan Talents Program Supported by Qingdao National Laboratory for Marine Science Technology (Grant no. 2015ASTP), the Chinese Academy of Sciences Strategic Priority Project, the Western Pacific Ocean System (Grant nos. XDA11010105 and XDA11020306), Taishan Scholarship, the Recruitment Program of Global Experts, Qingdao Innovative Program (Grant no. 13-CX-22) and Shandong Independent Innovation Major Program for key technology (2014GJJS0101).

Open Access This article is distributed under the terms of the Creative Commons Attribution 4.0 International License (http://creativecommons.org/licenses/by/4.0/), which permits unrestricted use, distribution, and reproduction in any medium, provided you give appropriate credit to the original author(s) and the source, provide a link to the Creative Commons license, and indicate if changes were made.

\section{References}

Birgin EG, Martinez JM, Raydan M (2000) Nonmonotone spectral projected gradient methods on convex sets. Siam J Optim 10:1196-1211

Bjerknes J (1969) Atmospheric teleconnections from equatorial Pacific. Mon Weather Rev 97:163-172

Blumenthal MB (1991) Predictability of a coupled ocean atmosphere model. J Clim 4:766-784

Chen D, Zebiak SE, Busalacchi AJ, Cane MA (1995) An improved procedure for EI Niño forecasting: implications for predictability. Science 269:1699-1702

Chen D, Cane MA, Kaplan A, Zebiak SE, Huang D (2004) Predictability of El Niño over the past 148 years. Nature 428:733-736

Duan WS, Wei C (2012) The spring predictability barrier for El Nino events and its possible mechanism results from a fully coupled model. Int J Climatol 33:1280-1292

Duan WS, Zhang R (2010) Is model parameter error related to spring predictability barrier for El Nino events? Adv Atmos Sci 27:1003-1013

Duan WS, Xue F, Mu M (2009) Investigating a nonlinear characteristic of El Niño events by conditional nonlinear optimal perturbation. Atmos Res 94:10-18

Duan WS, Yu YS, Xu H, Zhao P (2012) Behaviors of nonlinearities modulating the El Niño events induced by optimal precursory disturbances. Clim Dyn 40:1399-1413

Gao C, Zhang R-H (2017) The roles of atmospheric wind and entrained water temperature (Te) in the second-year cooling of the 2010-12 La Niña event. Clim Dyn:1-21

Gao C, Wu XR, Zhang R-H (2016) Testing a four-dimensional variational data assimilation method using an improved intermediate coupled model for ENSO analysis and prediction. Adv Atmos Sci 33:875-888

Goswami BN, Shukla J (1991) Predictability of a coupled ocean atmosphere model. J Clim 4:3-22

Jin EK et al (2008) Current status of ENSO prediction skill in coupled ocean-atmosphere models. Clim Dyn 31:647-664

Kalnay E, Kanamitsu M, Kistler R et al (1996) The NMC/NCAR reanalysis project. Bull Am Meteorol Soc 77:437-471

Luo JJ, Masson S, Behera SK, Yamagata T (2008) Extended ENSO predictions using a fully coupled ocean-atmosphere model. J Clim 21:84-93

Moore AM, Kleeman R (1996) The dynamics of error growth and predictability in a coupled model of ENSO. Q J R Meteorol Soc 122:1405-1446

Mu M, Duan WS, Wang B (2003) Conditional nonlinear optimal perturbation and its applications. Nonlinear Proc Geophys 10:493-501

Mu M, Xu H, Duan WS (2007) A kind of initial errors related to "spring predictability barrier" for El Niño events in Zebiak-Cane model. Geophys Res Lett 34:L03709. doi:10.1029/2006GL027412

Mu M, Duan WS, Wang Q, Zhang R (2010) An extension of conditional nonlinear optimal perturbation and its applications. Nonlinear Process Geophys 17:211-220

Mu M, Yu YS, Xu H, Gong TT (2014) Similarities between optimal precursors for ENSO events and optimally growing initial errors in El Nio predictions. Theor Appl Climatol 115:461-469

Philander SGH (1983) El-Niño Southern oscillation phenomena. Nature 302:295-301

Reynolds RW, Smith TM (1994) Improved global sea surface temperature analyses using optimum interpolation. J Clim 7(6):929-948

Reynolds RW, Rayner NA, Smith TM, Stokes DC, Wang WQ (2002) An improved in situ and satellite SST analysis for climate. J Clim 15:1609-1625 
Tao L-J, Zhang R-H, Gao C (2017) Initial error-induced optimal perturbations in ENSO predictions, as derived from an intermediate coupled model. Adv Atmos Sci 34(6):791-803. doi:10.1007/ s00376-017-6266-4

Wang Q, Mu M (2015) A new application of conditional nonlinear optimal perturbation approach to boundary condition uncertainty. J Geophys Res Oceans 120:7979-7996

Wang CZ, Picaut J (2004) Understanding ENSO physics-a review. Geophys Monogr Ser 147:21-48

Xu H (2006) Studies of predictability problems for Zebiak-Cane ENSO model. Ph. D. Dissertation, Institute of Atmospheric Physics, Chinese Academy of Sciences

Xue Y (1997) Predictability of a coupled model of ENSO using singular vector analysis. Part I: Optimal growth in seasonal background and ENSO cycles. Mon Weather Rev 125:2043

Yu Y, Mu M, Duan WS (2012a) Does model parameter error cause a significant spring predictability barrier for El Nino events in the Zebiak-Cane model. J Clim 25:1263-1277

Yu Y, Mu M, Duan WS, Gong T (2012b) Contribution of the location and spatial pattern of initial error to uncertainties in El Nio predictions. J Geophys Res 117:203

Zebiak SE, Cane MA (1987) A model el nin-southern oscillation. Mon Weather Rev 115:2262-2278
Zhang R-H, Gao C (2016a) Role of subsurface entrainment temperature $(\mathrm{Te})$ in the onset of El Niño events, as represented in an intermediate coupled model. Clim Dyn 46(5-6):1417-1435

Zhang R-H, Gao C (2016b) The IOCAS intermediate coupled model (IOCAS ICM) and its real-time predictions of the 2015-16 El Niño event. Sci Bull 66(13):1061-1070. doi:10.1007/ s11434-016-1064-4

Zhang R-H, Gao C (2017) Processes involved in the second-year warming of the 2014-15 El Niño event as derived from an intermediate ocean model. Sci China Earth Sci 60(9):1601-1613. doi:10.1007/ s11430-016-0201-9

Zhang R-H, Zebiak SE, Kleeman R, Keenlyside N (2003) A new intermediate coupled model for El Niño simulation and prediction. Geophys Res Lett 30(19). doi:10.1029/2003GL018010

Zhang R-H, Zebiak SE, Kleeman R, Keenlyside N (2005) Retrospective El Niño forecasts using an improved intermediate coupled model. Mon Weather Rev 133:2777-2802

Zhang R-H, Fei Z, Jiang Z, Wang Z (2013) A successful real-time forecast of the 2010-11 La Niña event. Sci Rep 3:1108. doi:10.1038/ srep01108

Zhu J, Huang B, Marx L, Iii JLK, Balmaseda MA, Zhang R-H, Hu ZZ (2012) Ensemble ENSO hindcasts initialized from multiple ocean analyses. Geophys Res Lett 39:9602-9608 